\title{
The effect of neural density and contactor surround on vibrotactile
} sensation magnitude*

\author{
RONALD T. VERRILLO $\dagger+\dagger$ and STEVEN C. CHAMBERLAIN \\ Laboratory of Sensory Communication \\ Syracuse University, Syracuse, New York 14627
}

Subjective magnitude characteristics for vibrotaction were determined by the method of numerical magnitude balance for three body sites varying in the density of neural innervation: the distal pad of the middle finger, the thenar eminence, and the volar forearm. The effects of a rigid surround upon threshold and the rate of growth of subjective intensity were measured. The results support the suggestion that absolute threshold and the rate of growth of subjective intensity are inverse functions of the total number of sensory neural units stimulated rather than being related simply to the density of neural innervation.

The specification of the physical stimulus has always been a central problem in the study of sensory systems. For vision and hearing, the effect upon sensation of many stimulus parameters is known so that adequate controls can be provided. Over the past several decades, interest in the use of the skin as an additional channel of communication and the trend toward seeking common functional characteristics among the sensory systems have provided the impetus for efforts to examine more closely the parameters essential to mechanoreception. A number of physical parameters important in the determination of the threshold of detectability have been investigated. These include: frequency (Békésy, 1939; Sherrick, 1953; Verrillo, 1963), contactor size (Verrillo, 1963, 1966; Craig, 1968; Craig \& Sherrick, 1969), static penetration of the contactor (Babkin et al, 1961; Verrillo, 1962; Craig \& Sherrick, 1969), a number of temporal parameters (Verrillo 1965), and the site of stimulation on the body (Verrillo, 1968), to name a few. The number of studies concerning these parameters at suprathreshold levels of stimulation is considerably smaller, being limited largely to frequency (Stevens, 1959, 1968; Verrillo et al, 1969) and body site (Stevens, 1959; Békésy, 1957, 1958, 1959). The present study is concerned

*The study was supported by Grant GB-8412 from the National Science Foundation and G r a n t NINDB-NB-03950-08 from the National Institutes of Health, U.S. Department of Health, Education and Welfare.

+Address: Laboratory of Sensory Communication, Syracuse University, 821 University Avenue, Syracuse, New York 13210.

+The senior author is at present a NATO Senior Fellow in Science at the Department of Human Anatomy, University of Oxford, England. in part with the effect of body site on the growth of vibrotactile sensation.

Békésy $(1957,1959)$ noted that the magnitude of vibrotactile sensation grows more rapidly over areas of the body that have a low density of neural innervation (arm, cheek) than over areas more richly endowed with nerve endings (hand, lip). He attributed this difference to the funneling action of the nervous system (Békésy, 1958). Stevens (1959) tested the fingers and forearm and found that, whereas the slope of the subjective magnitude function near threshold was indeed steeper for the arm, the slope at higher intensities was not very different for the two sites.

A stimulus condition that is often left uncontrolled in vibrotactile experiments is the spread of vibration over the surface of the skin. It is possible to limit the vibratory disturbance to the immediate vicinity of the contactor by the use of a rigid surround which leaves a gap of free skin only slightly larger than the contactor itself (Eijkman \& Vendrick, 1960; Verrillo, 1963). The effect of varying amounts of free skin surrounding the vibrating contactor is known for threshold measurements (Verrillo, 1962), but at suprathreshold levels of stimulation the effect of the presence or absence of a rigid surround is unknown. To study the effect upon subjective magnitude functions of a rigid surround is a second major aim of the present investigation.

\section{APPARATUS AND METHOD}

The apparatus and general experimental procedures have been described in detail in earlier publications (Verrillo, Fraioli, \& Smith, 1966; Verrillo, 1970). Briefly, the $S$ and vibrator are located inside $a$ booth that attenuates unwanted sounds and vibrations. The S's hand and forearm rest upon a rigid surface, with the vibrator situated on a platform below. The platform can be moved to adjust the height of the vibrator with respect to the surface of the skin. The same nine Ss participated in all experiments.

In experiments using a rigid surround, a disk containing a hole $2 \mathrm{~mm}$ larger than the diameter of the contactor was inserted into the rigid surface. The rigid surface surrounding the test sites was covered with a 1/8-in. pad of foam rubber to prevent the spread of vibrations that might be transmitted across the gap via the body tissues to other portions of the hand or arm. Removal of the surround permitted the free spread of vibrations over the surface of the skin. For testing the middle finger pad without surround, the forearm and the four fingers flanking the middle finger rested on the rigid platform. The middle finger was suspended free of contact except at the fingernail, which rested upon a lip protruding from the edge of the rigid surface. This arrangement was devised in order to provide a fixed position for the finger, thus maintaining a constant relationship between it and the height of the contactor. Careful testing showed that vibrations did not spread via the fingernail to other fingers resting on the rigid surface and foam pad surrounding the opening. Testing of the thenar eminence with a free

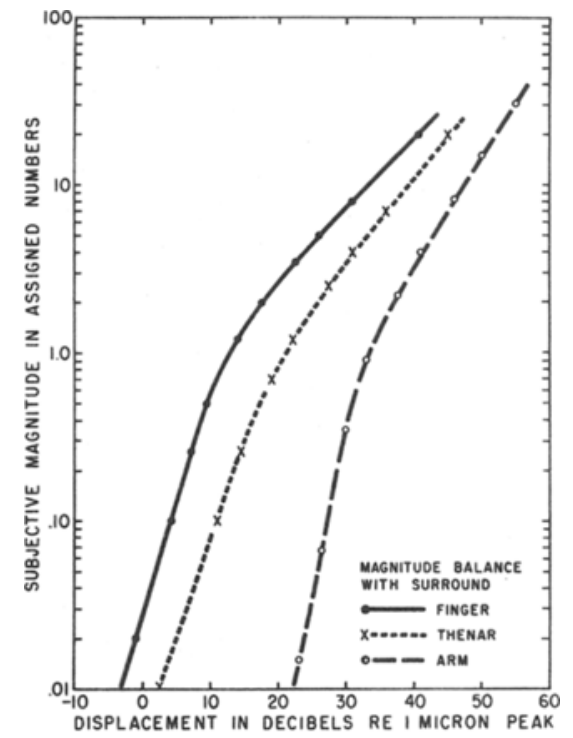

Fig. 1. Comparison of magnitude balance function obtained in this experiment (O) with that of an earlier experiment (O). Both functions were determined for a frequency of $250 \mathrm{~Hz}$ using a contactor area of $2.9 \mathrm{~cm}^{2}$ óver the thenar eminence. At medium and high intensities, the slope of both functions is $\mathbf{. 4 3 5}$. 


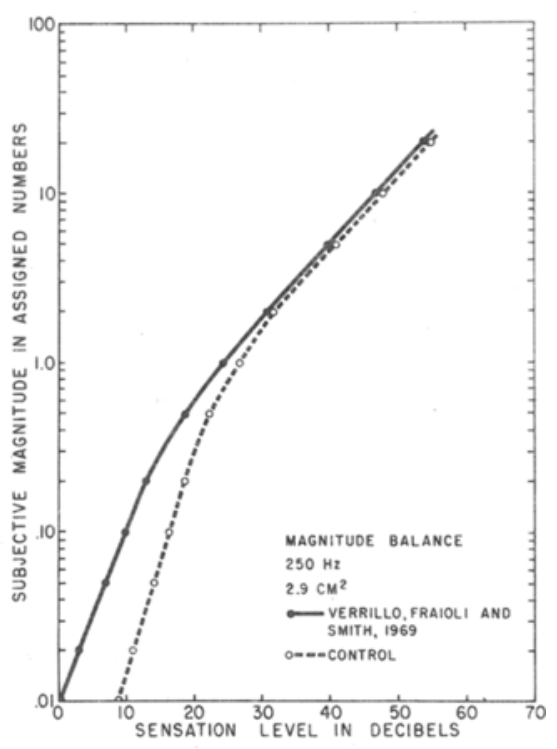

Fig. 2. Comparison of magnitude balance functions determined at the distal pad of the middle finger $(O)$, the thenar eminence $(x)$, and the volar forearm (O), using a rigid surround. Thresholds of detectability in decibels re 1 micron of peak displacement are: finger, $-10 \mathrm{~dB}$; thenar eminence, $-6 \mathrm{~dB}$; and forearm, $+17 \mathrm{~dB}$. Measurements were made using a frequency of $250 \mathrm{~Hz}$ and a contactor area of $.28 \mathrm{~cm}^{2}$

surround was accomplished by having the $S$ rest his forearm up to the wrist on the rigid platform. The hand was free of contact up to the tips of his fingers, which rested on the platform across the gap. Measurements taken on the forearm with a free surround were obtained with the forearm free of contact between the medial epicondyle and the wrist. Measurements were made at the distal pad of the middle finger, the center of the thenar eminence, and $8 \mathrm{~cm}$ distal to the medial epicondyle on the volar forearm.

The size of the contactor was $.28 \mathrm{~cm}^{2}$. A $250-\mathrm{Hz}$ sine wave was used with a temporal sequence of $900 \mathrm{msec}$ on and an interstimulus interval of $600 \mathrm{msec}$. The rise-fall time was 50 msec. The vibrator was adjusted in all experiments so that the contactor was raised $0.5 \mathrm{~mm}$ beyond minimum contact with the skin.

Subjective magnitude functions were determined by the method of numerical magnitude balance (Hellman \& Zwislocki, 1963), which is the nonnormalized combination of the methods of magnitude estimation and magnitude production (Stevens, 1955, 1958). The procedure is described in detail by Verrillo et al, 1969. Three measurements were made at each suprathreshold level. The first run was discarded and Measurements 2 and 3 were averaged for each S. Magnitude estimation and magnitude production curves were obtained by geometric averaging of the responses of the nine Ss. The results of magnitude estimation and magnitude production were then averaged (geometric mean) from group data to produce the magnitude balance function. Thus, each data point on the magnitude balance curves represents 36 measurements. Threshold measurements are reported in decibels referred to 1.0 micron of peak displacement.

It should be noted that the slopes of the subjective magnitude curves have been computed in terms of intensity rather than amplitude of displacement, which is a departure from current practice. The departure is made for several reasons. Research has shown that near the threshold of detectability there is a direct proportionality between the intensity of the stimulus and neural activity (Zwislocki, 1969; Zwislocki et al, 1969). Furthermore, intensity values permit a direct comparison between different sense modalities. Intensity may be used across all sensory systems, whereas a mplitude is limited to

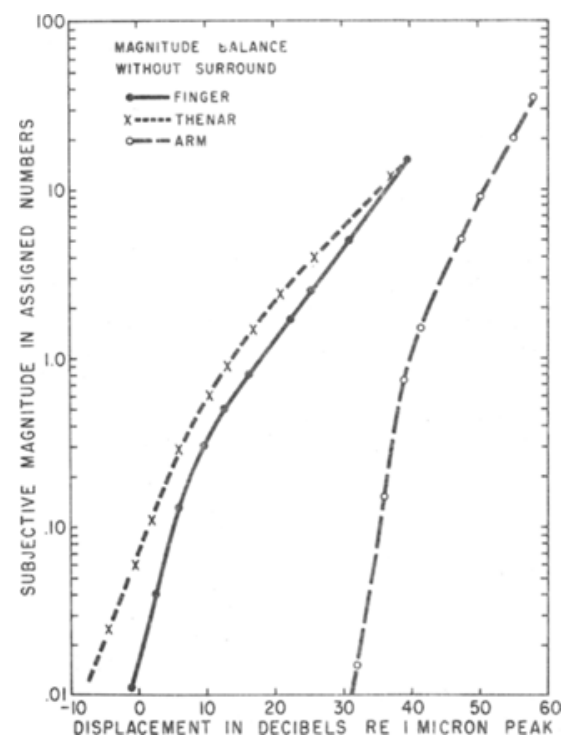

Fig. 3. Comparison of magnitude balance functions determined at the distal pad of the middle finger $(O)$, the thenar eminence $(x)$, and the volar forearm (O). Thresholds of detectability in decibels re 1 micron of peak displacement are: thenar eminence, $-14 \mathrm{~dB}$; finger, $-9.5 \mathrm{~dB}$; and forearm, +27.5 dB. Measurements were made without a rigid surround at a frequency of $250 \mathrm{~Hz}$ using a contactor area of $.28 \mathrm{~cm}^{2}$.

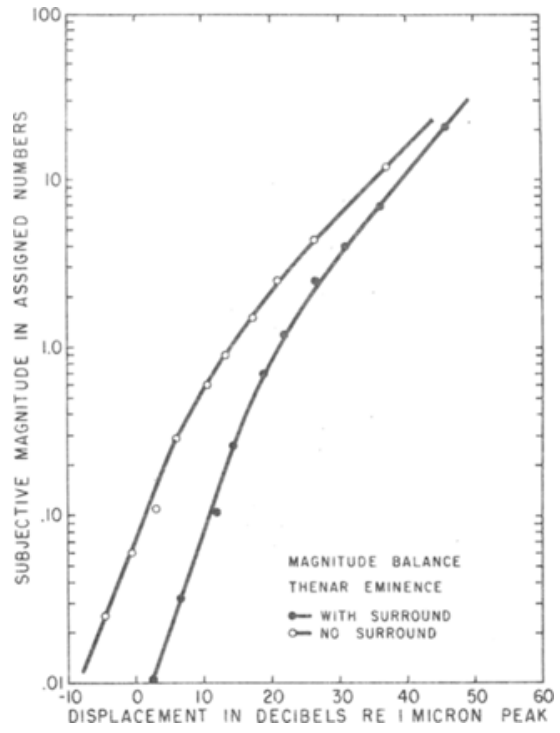

Fig. 4. Magnitude balance functions determined over the thenar eminence with (O) and without (O) a rigid surround. Removal of the rigid surround resulted in an increase of sensitivity and a decreased growth rate of subjective intensity. The slopes of the power functions are .5y with surround and .40 without surround.

mechanoreceptive systems. Direct comparisons of these slopes with earlier results may be made by halving the slope values reported in terms of amplitude (Verrillo et al, 1969; Verrillo, 1970).

\section{RESULTS}

\section{Control Experiment}

Prior to performing the main experiments, it was decided to repeat the experimental conditions of an earlier experiment (Verrillo et al, 1969; Verrillo, 1970) as a test of the procedures. Magnitude balance functions were determined at a frequency of $250 \mathrm{~Hz}$ with a $2.9-\mathrm{cm}^{2}$ contactor, using procedures and experimental conditions identical to those used before. The only modification was an improvement in the stability of the rigid platform upon which the S's hand and arm rested.

The results of the control experiment are presented in Fig. 1 along with comparison data obtained from six Ss in the earlier experiment. Comparison of the magnitude balance curves measured over the thenar eminence shows that the two groups yielded identical power functions $(0.435)$ in the upper portion of the curve. Near threshold the current group of Ss produced a slightly steeper function with a slope of 1.3 as compared to a slope of 1.0 for the earlier data.

The reason for this difference is not 


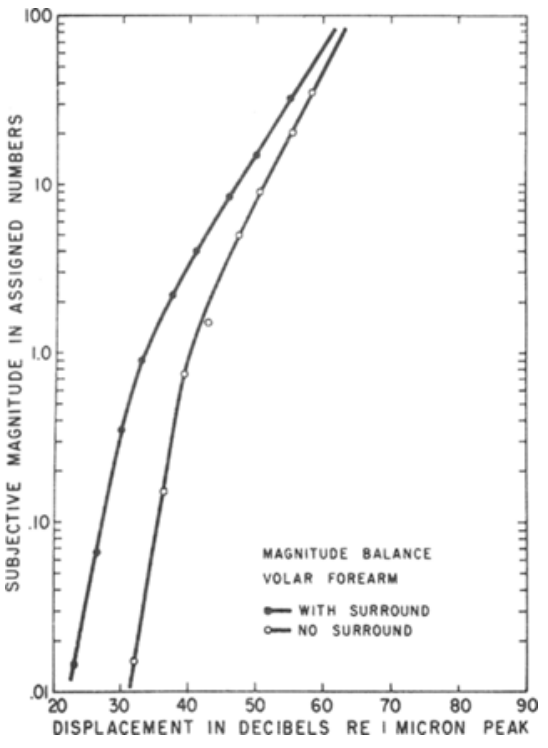

Fig. 5. Magnitude balance functions determined over the volar: forearm with (O) and without (O) a rigid surround. Removal of the surround resulted in a decrease of sensitivity and an increased growth rate of subjective intensity. The slopes of the power functions are .65 with surround and .77 without surround.

entirely clear, but it may be related to the improvements that were made in the rigidity of the platform. The close correspondence between the two sets of data was accepted as evidence of the reliability of the testing procedures used in the present experiments.

Experimental Results

When the rigid surround was used (Fig. 2), the slopes of sensation magnitude became progressively steeper from the finger (.45), to the thenar eminence (.50), to the forearm (.65). The absolute thresholds measured at these sites were $-10,-6$, and $+17 \mathrm{~dB}$, respectively. The growth of vibrotactile sensation appeared to be an inverse function of neural density and of the threshold of detectability. The order of slope value and body site shifted when the rigid surround was removed (Fig. 3). The sequence now was thenar eminence (.40), finger (.48), and forearm (.77). The slopes are no longer related to neural density, but again they are inversely related to absolute thresholds, which were $-14,-9.5$, and $+27.5 \mathrm{~dB}$, respectively, for thenar, finger, and forearm.

The slopes obtained at each body site with and without surround are compared in Figs. 4, 5, and 6. In each instance, the condition of surround that resulted in a higher threshold also produced a steeper sensory magnitude function.
The slope obtained at the thenar eminence with surround $(.50)$ differed from that of the control experiment $(.435)$, although the site and surround conditions were the same. This may have been due to the different contactor sizes used in the two procedures. The contactor area was $2.9 \mathrm{~cm}^{2}$ in the control experiment so that the results could be compared to a previous experiment. In the experiment proper, the area used was $.28 \mathrm{~cm}^{2}$, which constitutes a considerable reduction in size. The effect of contactor size on the threshold detectability is well known (Verrillo, 1963; Craig, 1968; Craig \& Sherrick, 1969), and the relationship between thresholds and slopes of subjective intensity reported above indicate that contactor size is the most likely reason for the discrepancy of experimental and control data for the thenar eminence.

\section{DISCUSSION}

Threshold Measurement

The threshold levels were correlated with the density of neural innervation in the expected direction of greater sensitivity as density increased from arm to thenar and finger. This relationship was seen, however, only when the area of stimulation was carefully controlled by the use of the rigid surround. The effect upon the threshold of detectability of surround vs no surround was different for the three regions tested. Over the thenar eminence, the effect of removing the surround was predictable; the free skin allowed the vibratory disturbance to propagate over a large area of skin and thus stimulated a greater number of sensory units. The effects of spatial summation may be observed in the threshold shift from $-6 \mathrm{~dB}$ with surround to $-14 \mathrm{~dB}$ without surround.

This effect was not seen, however, when the surround was removed from the finger pad. The threshold shift here was minor, from $-10 \mathrm{~dB}$ with surround to $-9.5 \mathrm{~dB}$ without surround. The reason for this small change is not entirely clear, but several factors may be suggested. First, the extent of additional free tissue available to stimulation when the surround is removed is proportionately more limited on the finger than over the thenar eminence. The effects of spatial summation are more pronounced when surface waves can propagate over a wide area. In addition, the spread of surface waves from the fingertip is toward regions of the hand that had a smaller concentration of neural units. Békésy (1959) has demonstrated that the interactions are complex between areas of unequal neural density, including shifts in localization and changes in subjective magnitude slope values. Removal of the surround increases the complexity of the physical and neural interactions. The effects of these interactions are poorly understood. The same complex interactions may account for the contradiction of a decrease in sensitivity from +17 to $27.5 \mathrm{~dB}$ when the surround was removed from the forearm.

\section{Sensation Magnitude}

The slope of the sensation magnitude curve is inversely related to the sensitivity of the region when the area of stimulation is carefully controlled by a rigid surround. This finding is consistent with observations by Békésy (1957, 1959). Since sensitivity is closely related to density of neural innervation, we concur with Békésy that regions of low neural density yield steeper slopes of subjective magnitude than do regions of high density. The finger pad, which has the lowest threshold with surround $(-10 \mathrm{~dB})$ and the highest neural density, also has the flattest curve (.45) among the three regions tested (Fig. 2).

The relationship of slope to neural density failed, however, when control over the area of stimulation was reduced by removing the rigid surround. Without the surround, the thenar region had the lowest threshold

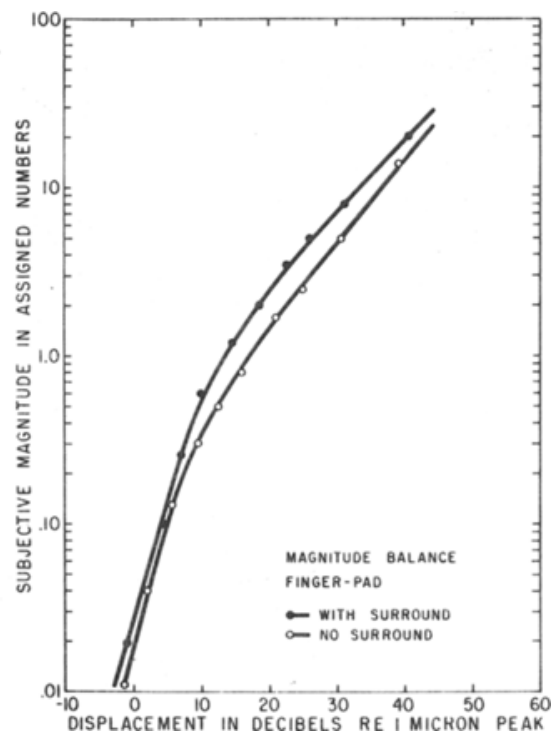

Fig. 6. Magnitude balance functions determined at the distal pad of the middle finger with (O) and without (O) a rigid surround. Removal of the surround produced a slight change in threshold and resulted in an increase in the slope of the power function from 45 with surround to .48 without surround. 
and also had the flattest slope of sensory magnitude $(.40)$, whereas the finger, which has the greatest neural density, produced a slope of .48 when the surround was removed (Fig. 3).

The single best correlate of slope magnitude appears to be the threshold of detectability regardless of body site, density of innervation, or condition of surround. The phenomenon can be seen with respect to body site with (Fig. 2) and without (Fig. 3) surround. It can also be observed at the same body site as the thresholds are altered by manipulating the surround condition (Figs. 4, 5, and 6). Furthermore, increases in the size of the contactor was accompanied by a decrease in the slope. At the thenar eminence a $2.9-\mathrm{cm}^{2}$ contactor yielded a slope of 0.435 and a .28- $\mathrm{cm}^{2}$ contactor resulted in a slope of 0.50 . This relationship is being systematically investigated. In every instance the slope varies directly with the absolute threshold. Conditions that produce high thresholds also yield steep slopes of sensory magnitude.

The results suggest that it is not neural density per se that determines the threshold level and the slope of the subjective magnitude function, but rather the number of sensory units activated by the stimulus. When the number of units activated is limited by confining the vibratory disturbance to a given area, the values for the threshold and the curve of subjective magnitude growth are inversely related to the density of neural innervation. This is because within a given area the number of sensory units increases as a function of neural density. When the vibration is allowed to spread unhampered to regions distant from the site of stimulation, the threshold and subjective magnitude values are still determined by the total number of neural units activated by the disturbance. This number may now be unrelated to the density of innervation, depending on the physical conditions of the region under investigation.

Spatial summation is certainly a sufficient explanation of the drop in threshold when a larger number of sensory units is activated. The underlying law which governs the changes in the slope of sensation magnitude is not known. This phenomenon has been suggested as the cutaneous analog of recruitment in hearing and similarly no theory has been advanced that will adequately explain why these events occur.

BABKIN $V$ PFERENCES TUMARKINA, L. N.. \& CHER NYAK, R. I. Investigations of vibration sensitivity and factors affecting it. Biophysics, 1961, $6,39-43$.

BEKESY, G. von. Ueber die Vibrationsempfindung. Akustische Zeitschrift, 1939, 4, 316-334.

BEKESY, G. von. Neural volleys and the simlarity between some sensations produced by tones and by skin vibrations. Journal of the Acoustical Society of America, 1957, 29, 1059-1069.

BÉKÉSY, G. von. Funneling in the nervous system and its role in loudness and sensation intensity on the skin. Journal of the Acoustical Society of America, 1958, 30, 399-412.

BÉKASY, G. von. Neural funneling along the skin and between the inner and outer hair cells of the cochlea. Journal of the Acoustical Society of America, 1959, 31 . 1236-1249.

CRAIG, J. C. Vibrotactile spatial summation. Perception \& Psychophysics, $1968,4,351-354$.

CRAIG, J. C., \& SHERRICK, C. E. The role of skin coupling in the determination of vibrotactile spatial summation. Perception \& Psychophysics, 1969, 6, 97-101.

EIJKMAN, E., \& VENDRICK, A. J. H. Dynamics of the vibration sense at low frequency. Journal of the Acoustical Society of America, 1960, 32, 1134-1139.

HELLMAN, R. P., \& ZWISLOCKI, J. Monaural loudness function at $1,000 \mathrm{cps}$ and interaural summation. Journal of the
Acoustical Society of America, 1963, 35, 856-865.

SHERRICK, C. E. Variables affecting sensitivity of the human skin to mechanical vibration. Journal of Experimental Psychology, 1953, 45, 273-282.

STEVENS, S. S. The measurement of loudness. Journal of the Acoustical Society of America, 1955, 27, 815-829.

STEVENS, S. S. Problems and methods of psychophysics. Psychological Bulletin. $1958,54,177-196$.

STEVENS, S. S. Tacile vibration: Dynamics of sensory intensity. Journal of Experimental Psychology, 1959, 57, 210-218.

STEVENS, S. S. Tactile vibration: Change of exponent with frequency. Perception \& Psychophysics, 1968, 3, 223-228.

VERRILLO, R. T. Investigation of some parameters of the cutaneous threshold for vibration. Journal of the Acoustical Society of America, 1962, 34, 1768-1773.

VERRILLO, R. T. Effect of contactor area on the vibrotactile threshold. Journal of the Acoustical Society of America, 1963, $35,1962-1966$.

VERRILLO, R. T. Temporal summation in vibrotactile sensitivity. Journal of the Acoustical Society of America, 1965, 37, 843-846.

VERRILLO, R. T. Effect of spatial parameters on the vibrotactile threshold. Journal of Experimental Psychology, $1966,71,570-575$.

VERRILLO, R. T. A duplex mechanism of mechanoreception. In $D$. $R$. Kenshalo (Ed.), The skin senses. Springfield: C C Thomas, 1968.

VERRILLO, R. T. Subjective magnitude functions for vibrotaction. I.E.E.E. Transactions on Man-Machine Systems, 1970, MMS-11, 19-24.

VERRILLO, R. T., FRAIOLI, A. J., \& SMITH, R. L. Sensation magnitude of vibrotactile stimuli. Perception \& Psychophysics, 1969, 6, 366, 372.

ZWISLOCKI, J. A generalized intensity characteristic of sensory receptors. Semiannual Research Report, Laboratory of Sensory Communication, Syracuse University, 1969, 10, 7-9.

ZWISL OCKI, J., ADAMS, W. B., BARLOW, R. B., \& KELTSKY, E. J. Intensity characteristics of sensory rece ptors. Third International Biophysics Congress of the International Union for Pure and Applied Biophysics, Cambridge, Mass., 1969, p. 262 (Abstract).

(Received for publication September 15 $1970)$. 